\title{
Floraciones de fitoplancton y variación de la estructura comunitaria fitoplanctónica en tres lagos someros eutróficos de Chile Central
}

\section{Phytoplankton blooms and changes in the phytoplankton community structure in three eutrophic shallow lakes of Central Chile}

\author{
Viviana Almanza ${ }^{1,2 *}$, Oscar Parra ${ }^{1}$, Carlos E. De M. Bicudo ${ }^{3}$, Mariela A. González ${ }^{4}$, Marcela \\ LOPEZ ${ }^{1} \&$ ROBERTO URRUTIA ${ }^{1,2}$ \\ ${ }^{1}$ Centro EULA-Chile, Universidad de Concepción, Barrio Universitario, Concepción, Chile. \\ ${ }^{2}$ Centro de Recursos Hídricos para la Agricultura y la Minería, Victoria 1295, Barrio Universitario, Concepción, Chile. \\ ${ }^{3}$ Instituto de Botânica, Av. Miguel Estéfano, 3687, 04301-012 São Paulo, SP, Brasil. \\ ${ }^{4}$ Departamento de Botánica, Universidad de Concepción, Casilla 160-C, Concepción, Chile. \\ *valmanza@udec.cl
}

\begin{abstract}
RESUMEN
La comunidad fitoplanctónica de tres lagos someros eutróficos ubicados en la misma zona geográfica fue comparada durante un periodo de tres años. Aunque estos lagos son similares en muchos aspectos (p.ej. ubicación, origen, profundidad media, alta concentración de nutrientes, influencia humana) desarrollan comunidades de fitoplancton muy diferentes. En Lo Galindo dominan Zygnematophyceae, Cyanophyceae y Chlorophyceae, en Lo Méndez Dinophyceae y Bacillariophyceae y en Las Tres Pascualas Chlorophyceae, Cryptophyceae y Euglenophyceae. En Lo Méndez y Lo Galindo se presentaron frecuentes floraciones de diferentes grupos de microalgas (cianobacterias, algas verdes, diatomeas y dinoflagelados), las que generaron aumentos de abundancia en diferentes épocas del año, incluso en invierno. En Las Tres Pascualas, las floraciones fueron poco frecuentes. La ocurrencia y frecuencia de floraciones de especies representantes de los grupos funcionales M, C, Y y Lo y morfológicos VII, VI, IV y V reflejan las condiciones eutróficas de los lagos, siendo indicadoras del grado de contaminación de estos sistemas que influencia su uso actual y futuro.
\end{abstract}

Palabras clave: Floraciones, comunidad de fitoplancton, grupos funcionales, estado trófico, lagos urbanos.

\begin{abstract}
The phytoplankton community of three eutrophic shallow lakes located in the same geographical area was compared over a period of three years. Although these lakes are similar in many respects (e.g. location, origin, average depth, high concentration of nutrients, human influence) develop very different phytoplankton communities. Lo Galindo is dominated by Zygnematophyceae, Cyanophyceae and Chlorophyceae, while in Lo Méndez the groups Dinophyceae and Bacillariophyceae and in Las Tres Pascualas the Chlorophyceae, Cryptophyceae and Euglenophyceae. In Lo Mendez and Lo Galindo are frequent blooms of different groups of algae (cyanobacteria, green algae, diatoms and dinoflagellates), which generated wealth increases at different times of the year, even in winter they were presented. In Las Tres Pascualas blooms were rare. The occurrence and frequency of blooms of species representatives of the M, C,Y and Lo functional groups, and VII, VI, IV and V morphological groups, reflect the eutrophic lakes, being indicative of the degree of contamination of these systems that influence their present and future use.
\end{abstract}

KEYwORDs: Blooms, phytoplankton, functional groups, eutrophication, urban lakes.

\section{INTRODUCCIÓN}

La eutrofización de los sistemas acuáticos es uno de los problemas ambientales actuales de mayor distribución a nivel mundial (Schindler 2015). El principal efecto de la eutrofización en estos sistemas es la formación de floraciones o "blooms" de microalgas producidas principalmente por los grupos Cyanophyceae (cianobacterias), Dinophyceae, Chlorophyceae, Cryptophyceae, Chrysophyceae, Euglenophyceae y Bacillariophyceae (Paerl 1988, Siguee 2005).

Las floraciones se expresan de diversa forma dependiendo 
de la especie y el cuerpo de agua (Siguee 2005), pueden ocurrir en la superficie y/o a profundidades específicas en la columna de agua, son favorecidas por diversas condiciones ambientales, como el exceso de nutrientes, la temperatura, alta intensidad de luz, $\mathrm{pH}$, estabilidad de la columna de agua (por ausencia de viento) (Robarts \& Zohary 1987, Jacoby et al. 2000, Davis et al. 2009, Xu et al. 2010). Procesos poblacionales como el aumento de tasa de crecimiento y capacidad de competir con otras algas o la reducción del pastoreo, dispersión, sedimentación y mortalidad, también están implicados en la formación y desarrollo de las floraciones (Imberger et al. 1983). En un ambiente con óptimas condiciones, se asimila toda la producción primaria (tasa de producción de biomasa fitoplanctónica), sin embargo, si la producción excede el consumo, el fitoplancton se acumula y puede llegar a ser visible generando cambios de color del agua (frecuentemente amarillo, verde, rojo o café) y/o de la textura del agua (Siguee 2005). En muchos casos el aumento de biomasa se transfiere a través de la red trófica contribuyendo al re-suministro de nutrientes en la columna de agua sin tener efectos adversos sobre el ambiente (Carstensen et al. 2007). Contraria a esta situación, la acumulación de biomasa genera diversos efectos negativos en los sistemas acuáticos y en los usos que se realizan sobre el recurso agua y el sistema en general, siendo consideradas un problema ambiental y de salud emergente para la población.

Las floraciones producen cambios en la red trófica y la productividad de los lagos, disminución de la transparencia del agua, disminución de la diversidad de especies, cambios en la red trófica, por la pérdida del hábitat (disminución en la transparencia del agua) (UNESCO 2009). A su vez, estos cambios generan una disminución del valor estético y paisajístico de los ecosistemas (por cambios en su coloración, acumulaciones de biomasa y mal olor), lo que afecta la calidad del agua impidiendo su utilización para la natación, navegación y demás actividades acuáticas (Reynolds 2006). Específicamente, las floraciones de cianobacterias pueden producir toxinas con propiedades hepatotóxicas, neurotóxicas o dermatotóxicas que afectan la salud humana y animal (Chorus \& Bartram 1999).

En las últimas décadas la frecuencia y la distribución global de las floraciones de fitoplancton, principalmente de cianobacterias tóxicas, ha aumentado a nivel mundial, lo que ha sido atribuido al efecto combinado de la eutrofización y el cambio del clima (Paerl 2014).

La identificación de los principales factores bióticos y abióticos que condicionan el aumento de biomasa de fitoplancton en los lagos es esencial para la adecuada gestión de los ecosistemas de aguas continentales (Peretyatko et al. 2007). Existen diferentes métodos de evaluación que implican desde la composición taxonómica y la diversidad de especies (Stevenson \& Smol 2003), hasta métodos de agrupación a partir de aspectos fisiológicos y/o morfológicos. Estos últimos permiten relacionar la presencia y el comportamiento de las comunidades algales con determinadas condiciones tanto físicas como químicas de los cuerpos de agua (Reynolds et al. 2002, Padisák et al. 2009) sin necesariamente hacer mediciones en el ambiente y/o donde el conocimiento de estos parámetros es escaso (Kruk et al. 2010). Los enfoques basados en las características funcionales de las especies han sido cada vez más aplicados para explicar y predecir la respuesta de las especies de fitoplancton a las condiciones ambientales (Kruk \& Segura 2012).

Desde hace décadas, los lagos someros Lo Galindo, Las Tres Pascualas y Lo Méndez, ubicados en la ciudad de Concepción, reciben altas cargas de nutrientes desde la cuenca urbana (Almanza et al. 2016a). Los efectos de la contaminación por nutrientes han sido observados con la mortandad masiva de peces (Parra et al. 1986, Parra 2009), presencia de floraciones de cianobacterias y dinoflagelados (Parra et al. 1981, Campos et al. 2005, Almanza et al. 2016b) y crecimiento masivo de macrófitas (Parra 2009). Lo que se ha traducido en una disminución del valor estético y recreativo de estos cuerpos de agua, restringiendo las alternativas de uso y limitando las actividades que los ciudadanos pueden hacer en torno a estos sistemas.

Los objetivos de este trabajo fueron caracterizar y comparar las comunidades de fitoplancton de los tres lagos someros eutróficos y analizar las condiciones ambientales que influencian las variaciones espaciales y temporales de estas comunidades, específicamente en relación con las floraciones de fitoplancton.

\section{MATERIALES Y MÉTODOS}

Área DE ESTUdio

Los lagos Lo Méndez (LM) ( $36^{\circ} 48^{\prime} 03^{\prime \prime} \mathrm{S}$ y $\left.73^{\circ} 03^{\prime} 03^{\prime \prime} \mathrm{O}\right)$, Lo Galindo (LG) $\left(36^{\circ} 48^{\prime} 01^{\prime \prime} \mathrm{S}\right.$ y $\left.73^{\circ} 02^{\prime} 31^{\prime \prime} \mathrm{O}\right)$ y Las Tres Pascualas (LTP) $\left(36^{\circ} 48^{\prime} 50^{\prime \prime} \mathrm{S}\right.$ y $\left.73^{\circ} 02^{\prime} 30^{\prime \prime} \mathrm{O}\right)$ están ubicados en la ciudad de Concepción, Región del Biobío, Chile Central (Fig. 1). Son lagos poco profundos (profundidad promedio de $3 \mathrm{~m}$ ) de origen fluvial y no presentan estratificación térmica en verano. Las principales características morfométricas de estos lagos se resumen en la Tabla I.

\section{Métodos de MUeSTReo}

En cada uno de los lagos se tomaron muestras integradas de la columna de agua a dos profundidades (superficie y fondo) con una botella Niskin vertical de $1.5 \mathrm{~L}$ de capacidad. El muestreo fue realizado en forma mensual o bimensual abarcando todas las estaciones del año (verano, otoño, invierno y primavera), durante un periodo de tres años (2012-2014). Dependiendo de la superficie del cuerpo de agua se establecieron una o dos estaciones de muestreo. Parámetros de calidad del agua como temperatura $\left({ }^{\circ} \mathrm{C}\right)$, 
$\mathrm{pH}$, conductividad $\left(\mu \mathrm{S} \mathrm{cm} \mathrm{cm}^{-1}\right)$, oxígeno disuelto (mg $\left.\mathrm{L}^{-1}\right)$ fueron medidos "in situ" con una sonda multiparamétrica (Hydrolab modelo Quanta) 'in situ' en la columna del agua y la transparencia (m) con un disco Secchi. Para parámetros como fósforo total y ortofosfato $\left(\mathrm{mg} \mathrm{L}^{-1}\right)$ y los compuestos de nitrógeno (N-total, nitrato, nitrito y amonio) (mg L $\left.\mathrm{L}^{-1}\right)$ se tomaron muestras en frascos adecuados al análisis y se trasladaron en frío $\left(4^{\circ} \mathrm{C}\right)$ para su posterior análisis por espectrofotometría de absorción molecular y/o cromatografía iónica descritos en APHA (2012); fósforo total (4500 P), ortofosfato (4110 P), nitrógeno total (4500$\mathrm{N} \mathrm{C}$ ), nitrato $\left(4110-\mathrm{NO}_{3}\right)$, nitrito $\left(4110-\mathrm{NO}_{2}\right)$ y amonio $\left(4500-\mathrm{NH}_{4}\right)$. La determinación de la clorofila- $a$ se realizó filtrando $250 \mathrm{ml}$ de muestra, el filtro fue incorporado en un frasco plástico oscuro con $20 \mathrm{ml}$ de acetona al 90\%, la lectura se realizó $24 \mathrm{~h}$ después para la extracción total del compuesto. La determinación se realizó mediante filtración

TABla I. Características morfométricas de los lagos urbanos de Concepción. HE = Hipereutrófico.

TABLE I. Morphometric characteristics of Concepción urban lakes. HE = Hypereutrophic.

\begin{tabular}{lccc}
\hline \multicolumn{1}{c}{ LAGO $/$ PARÁMETRO } & LG & LTP & LM \\
\hline Area superficial $\left(\mathrm{m}^{2}\right)$ & 49.500 & 77.600 & 42.100 \\
Profundidad media $(\mathrm{Z})$ & 1,3 & 4,4 & 3,7 \\
Profundidad máxima $\left(\mathrm{Z}_{\mathrm{m}}\right)$ & 3,4 & 7,9 & 6,5 \\
Volumen $(\mathrm{V})\left(\mathrm{m}^{3}\right)$ & 62.500 & 344.500 & 157.800 \\
Máximo tiempo de residencia (días) & 30 & 30 & 30 \\
Estado trófico & $\mathrm{HE}$ & $\mathrm{HE}$ & $\mathrm{HE}$ \\
\hline
\end{tabular}

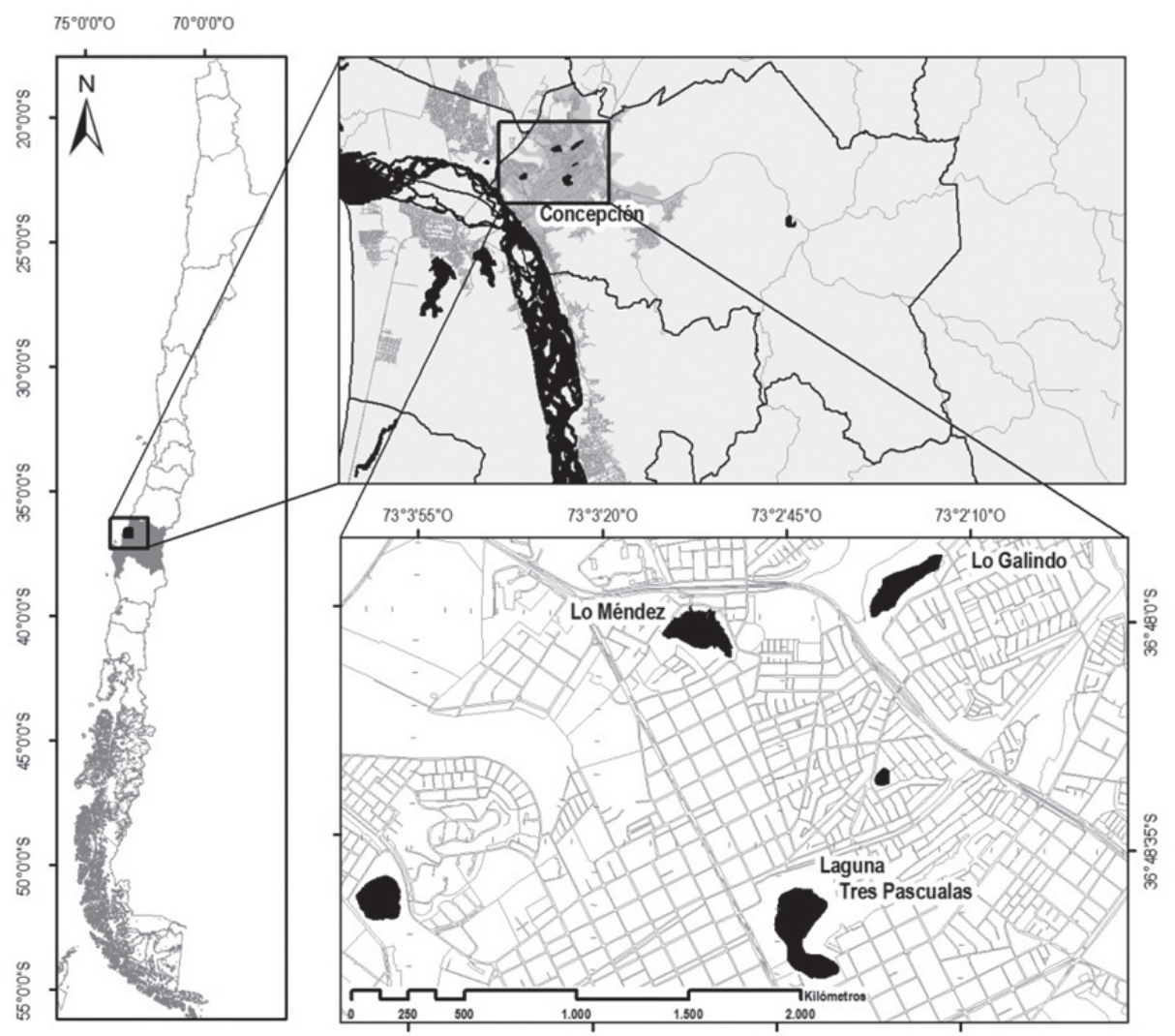

Figura 1. Lagos someros eutróficos de Lo Méndez, Lo Galindo y Las Tres Pascualas, Región del Biobío, Chile.

Figure 1. The shallow eutrophic lakes Lo Mendez, Lo Galindo and Las Tres Pascualas, Biobío Region, Chile. 
por membrana, con un fluorómetro 10-AU Turner Designs (Welschmeyer 1994).

Las muestras de fitoplancton fueron fijadas y preservadas en solución acuosa de lugol al 1\% y se cuantificó el número de células de microalgas por el método de Utermöhl (1958), a partir de cámaras de sedimentación de volumen conocido en un microscopio invertido (Zeiss Axiovert 35). La cuantificación de células se realizó por el conteo de campos (con aumento 40x) de observación escogidos al azar, su cantidad dependió de la frecuencia de las especies presentes en la muestra. En cada campo se contabilizaron todas las células encontradas, un total de 100 células de la especie más frecuente y/o un mínimo 400 por muestra (Elosegi \& Sabater 2009). En el caso de las cianobacterias (p.ej. Microcystis), las colonias fueron desintegradas por el método de hidrólisis alcalina (a $1 \mathrm{ml}$ de muestra se añadió 2 a 3 gotas de $\mathrm{KOH} 1 \mathrm{M}$ y se llevó a baño de maría a 90 ${ }^{\circ} \mathrm{C}$ por 45 min aproximadamente), el número de células se contabilizó en una cámara de Neubauer. Los resultados fueron reportados a través del número de células por litro (cél L ${ }^{-1}$ ) (Hötzel \& Croome 1999).

Para la identificación taxonómica se recogió material de la columna de agua con una red de plancton de $20 \mu \mathrm{m}$ de apertura de malla, una porción de la muestra fue fijada con lugol $1 \%$ y la otra mantenida en fresco. La identificación de los organismos se realizó hasta el nivel taxonómico más bajo posible, lo anterior se realizó mediante el uso de literatura especializada. Para la clasificación e identificación de las algas verdes se utilizó Bock et al. (2011), Leliaert et al. (2012) y Krienitz \& Bock (2012), para Cyanophyceae Komárek \& Anagnostidis (2000, 2005), Komárek (2013), Komárek et al. (2014), Chrysophyceae y Synurophyceae Andersen (2007), Cryptophyceae Cerino \& Zingone (2007) y Choi et al. (2013), Dinophyceae Moestrup \& Daugbjerg (2007), Euglenophyceae Marin et al. (2003) y Triemer \& Farmer (2007), para Bacillariaceae se utilizó Rumrich et al. (2000). Finalmente para todos los grupos algales se consultó Parra et al. (1982-1983) y Guiry \& Guiry (2016). Para facilitar la identificación taxonómica de diatomeas y dinoflagelados se observaron muestras en microscopia electrónica, se realizaron tinciones con tinta china y azul de metileno para observar el mucílago y las paredes transversales de filamentos en las cianobacterias. Se separaron las placas de los dinoflagelados con hipoclorito de sodio y se consultó con expertos para la confirmación taxonómica de las especies, incluyendo euglenas y algas.

ANÁLISIS DE DATOS

Todos los datos tomados fueron agrupados por lago y estación del año y analizados con estadística descriptiva (mínimo, máximo, promedio).

La comunidad de fitoplancton se describió mediante la identificación de todos los taxones de la comunidad a nivel de clase y determinaron diferentes índices biológicos como la abundancia relativa, riqueza de taxa (índice de Margalef, d) y diversidad (índice Shannon Wiener, H'). Además, los taxones que representaron más del $5 \%$ de la abundancia total de fitoplancton en cada lago, se clasificaron en grupos funcionales según la clasificación funcional de especies (FGs) propuesto por Reynolds et al. (2002) y modificado por Padisák et al. (2009) y la clasificación basada en la morfología (MBFGs) propuesta por Kruk et al. (2010).

Con el fin de determinar diferencias y similitud entre las comunidades presentes en y entre lagos y a nivel temporal, se realizó un análisis de conglomerados y de ordenamiento multidimensional no-métrico (MDS). Previamente, los datos de abundancia fueron transformados a $\log (n+1)$ y se utilizó el índice de Bray Curtis (Clarke \& Warwick 2001). Diferencias significativas entre los lagos y las estaciones del año fueron determinadas con el análisis ANOSIM a dos vías. Se identificaron los taxones que determinaron diferencias entre los lagos estudiados con el análisis de SIMPER. Los datos fueron analizados usando el programa PRIMER. Se identificaron los parámetros ambientales que mejor explican el patrón de la comunidad fitoplanctónica en cada lago mediante un análisis de correspondencia canónica CCA (longitud del gradiente fue ca. 2.9 SD) en el programa R.

\section{RESULTADOS}

VARIACIÓN ESPACIO-TEMPORAL DE LA COMUNIDAD FITOPLANCTÓNICA

Un total de 131 taxones de 12 clases fueron identificados en la composición del fitoplancton de los tres lagos estudiados (Fig. 2a). Los taxones registrados en cada cuerpo de agua se listan en la Tabla II. Un 61\% de los taxones reportados para todos los lagos se encuentran presentes en los tres sistemas estudiados.

Las microalgas más abundantes se distribuyeron en 15 diferentes FGs (Fig. 2b). Los grupos M, C, Y y L fueron los más importantes en términos de biomasa, los que están representados por especies que habitan ambientes eutróficos a mesotróficos como Microcystis aeruginosa (Kützing) Kützing (Codón M), Asterionella formosa Hassall (Codón C), Cryptomonas ovata Ehrenberg y Phacus sp. (Codón Y), Ceratium furcoides (Levander) Langhans (Codón $\mathrm{L}_{\mathrm{o}}$ ). Según la clasificación morfológica de Kruk et al. (2010), se determinaron cinco principales MBFGs (VII, VI, IV, V y I) (Fig. 2c).

La estructura de la comunidad fitoplanctónica fue diferente para cada lago, los resultados del análisis de conglomerados y del análisis multidimensional no métrico MDS mostraron que con un $20 \%$ de similitud se generan tres grupos que corresponden a cada uno de los lagos estudiados LTP (Las Tres Pascualas), LG (Lo Galindo) y LM (Lo Méndez) (Fig. 3 ), estas diferencias fueron estadísticamente significativas $\left(\right.$ ANOSIM $\left.\mathrm{R}_{\text {global }}=0,66 ; \mathrm{p}<0,05\right)$. 
Fitoplancton de lagos urbanos eutróficos, Chile central: Almanza, V. ET AL.

TABla II. Lista de taxa de microalgas encontradas en los lagos estudiados durante el periodo 2012-2014. (+) Presencia. FGs (C, E, F, J, K, Lo, M, P, Sn, T, Tb, Wi, X2, X1, Y), MBFGs (I, II, III, IV, V, VI e VII). LG: Lo Galindo, LM: Lo Méndez, LTP: Laguna Tres Pascualas.

TABLE II. List of taxa of microalgae found in the lakes studied during the period 2012-2014. (+) Presence. FGs (C, E, F, J, K, Lo, M, P, Sn, T, Tb, Wi, X2, X1, Y), MBFGs (I, II, III, IV, V, VI e VII). LG: Lo Galindo, LM: Lo Méndez, LTP: Laguna Tres Pascualas.

\begin{tabular}{|c|c|c|c|c|c|}
\hline TAXON & LG & LM & LTP & FGs & MBFGs \\
\hline \multicolumn{6}{|l|}{ CHRYSOPHYCEAE } \\
\hline Dinobryon divergens O.E.Imhof & & + & & $\mathrm{E}$ & II \\
\hline \multicolumn{6}{|l|}{ SYNUROPHYCEAE } \\
\hline Mallomonas sp. & & + & + & $\mathrm{E}$ & II \\
\hline Synura sp. & & + & & & \\
\hline \multicolumn{6}{|l|}{ CHLOROPHYCEAE } \\
\hline Acutodesmus acuminatus (Lagerheim) P.M.Tsarenko & + & + & + & $\mathrm{J}$ & IV \\
\hline Ankistrodesmus falcatus (Corda) Ralfs & & & + & & \\
\hline Ankistrodesmus spiralis (W.B.Turner) Lemmermann & + & & & & \\
\hline Ankyra judayi (G.M.Smith) Fott & & & + & XI & \\
\hline Chlamydomonas spp. & + & + & + & $\mathrm{X} 2$ & I \\
\hline Coelastrum microporum Nägeli & + & + & + & $\mathrm{J}$ & IV \\
\hline Coelastrum reticulatum (P.A.Dangeard) Senn & + & & & & \\
\hline Coelastrum sphaericum Nägeli & & & + & & \\
\hline Desmodesmus communis (E.Hegewald) E.Hegewald & + & + & + & & \\
\hline Desmodesmus opoliensis (P.G.Richter) E.Hegewald & + & & + & & \\
\hline Dimorphococcus sp. & & + & + & & \\
\hline Eudorina elegans Ehrenberg & + & + & + & & \\
\hline Kirchneriella sp. & + & & & & \\
\hline Monactinus simplex (Meyen) Corda & + & + & + & $\mathrm{J}$ & IV \\
\hline Monoraphidium contortum (Thuret) J.Komárková-Legnerová & + & & & & \\
\hline Monoraphidium griffithii (Berkeley) J.Komárková-Legnerová & + & & & & \\
\hline Monoraphidium irregulare (G.M.Smith) J.Komárková-Legnerová & & & + & & \\
\hline Monoraphidium minutum (Nägeli) J.Komárková-Legnerová & + & + & + & $\mathrm{XI}$ & IV \\
\hline Monoraphidium pusillum (Printz) J.Komárková-Legnorová & + & & & & \\
\hline Monoraphidium saxatile J.Komárková-Legnerová & + & + & + & $\mathrm{XI}$ & IV \\
\hline Pandorina sp. & + & & & & \\
\hline Pediastrum duplex Meyen & + & & & $\mathrm{J}$ & IV \\
\hline Pseudopediastrum boryanum (Turpin) E.Hegewald & + & & + & & \\
\hline Quadrigula closterioides (Bohlin) Printz & + & & & & \\
\hline Scenedesmus acutus Meyen & + & & & & \\
\hline Scenedesmus ecornis (Ehrenberg) Chodat & + & & & & \\
\hline Scenedesmus ovalternus Brébisson & + & & & & \\
\hline Selenastrum gracile Reinsch & + & & & & \\
\hline Sphaerocystis schroeteri Chodat & + & + & + & & \\
\hline Stauridium tetras Corda & + & + & & & \\
\hline Tetraëdron caudatum (Corda) Hansgirg & + & & & & \\
\hline Tetraëdron constrictum G.M.Smith & + & & & & \\
\hline Tetraëdron trigonum (Nägeli) Hansgirg & + & + & + & & \\
\hline Tetraspora lubrica (Roth) C.Agardh & + & & & & \\
\hline Volvox aureus Ehrenberg & + & + & + & & \\
\hline \multicolumn{6}{|l|}{ TREBOUXIOPHYCEAE } \\
\hline Botryococcus braunii Kützing & + & + & + & $\mathrm{F}$ & VII \\
\hline Chlorella spp. & + & & + & $\mathrm{X} 1$ & I \\
\hline Chodatella citriformis J.W. Snow & + & & & & \\
\hline
\end{tabular}




\begin{tabular}{|c|c|c|c|c|c|}
\hline TAXON & LG & LM & LTP & FGs & MBFGs \\
\hline Crucigeniella apiculata (Lemmermann) Komárek & + & + & + & $\mathrm{F}$ & \\
\hline Crucigeniella irregularis (Wille) P.M.Tsarenko \& D.M.John & + & & & & \\
\hline Dictyosphaerium sp. & + & & + & $\mathrm{F}$ & \\
\hline Heynigia sp. & & & + & & \\
\hline Hindakia sp. & & & + & & \\
\hline Nephrocytium agardhianum Nägeli & + & & & & \\
\hline Nephrocytium limneticum (G.M.Smith) G.M.Smith & & + & & & \\
\hline Oocystis lacustris Chodat & & + & + & $\mathrm{F}$ & VII \\
\hline Oocystis parva W.West \& G.S.West & + & & & $\mathrm{F}$ & VII \\
\hline \multicolumn{6}{|l|}{ ULVOPHYCEAE } \\
\hline Cladophora glomerata (Linnaeus) Kützing & & & + & & \\
\hline Ulothrix sp. & & & + & & \\
\hline \multicolumn{6}{|l|}{ ZYGNEMATOPHYCEAE } \\
\hline Closterium aciculare T.West & + & + & + & $\mathrm{P}$ & IV \\
\hline Closterium acuminatum Kützing ex Ralfs & + & & & & \\
\hline Closterium gracile Brébisson ex Ralfs & + & + & + & $\mathrm{P}$ & IV \\
\hline Closterium parvulum Nägeli & & & + & & \\
\hline Closterium praelongum var. brevius (Nordstedt) Willi Krieger & & & + & & \\
\hline Closterium setaceum Ehrenberg ex Ralfs & & & + & & \\
\hline Cosmarium depressum (Nägeli) P.Lundell & & + & & & \\
\hline Cosmarium pseudoprotuberans O.Kirchner & + & & & & \\
\hline Cosmarium subtumidum Nordstedt & + & & & & \\
\hline Gonatozygon kinahanii (W.Archer) Rabenhorst & & & + & & \\
\hline Micrasterias sp. & & + & + & & \\
\hline Mougeotia laetevirens (A.Braun) Wittrock & & + & & & \\
\hline Pleurotaenium sp. & & + & + & $\mathrm{J}$ & IV \\
\hline Spirogyra sp. & & & + & & \\
\hline Staurastrum chaetoceras (Schröder) G.M.Smith & + & & & & \\
\hline Staurastrum gracile Ralfs ex Ralfs & & & + & & \\
\hline Staurastrum orbiculare Meneghini ex Ralfs & + & & + & & \\
\hline Staurastrum pingue Teiling & + & + & + & $\mathrm{P}$ & IV \\
\hline Staurastrum sebaldi Reinsch var. sebaldi & + & + & & & \\
\hline Staurastrum tetracerum Ralfs ex Ralfs & + & & & & \\
\hline Staurodesmus indentatus (West \& G.S.West) Teiling & + & & & & \\
\hline Zygnema sp. & & & + & & \\
\hline \multicolumn{6}{|l|}{ CRYPTOPHYCEAE } \\
\hline Rhodomonas lacustris Pascher \& Ruttner & + & & + & & \\
\hline Cryptomonas erosa Ehrenberg & + & + & + & & \\
\hline Cryptomonas ovata Ehrenberg & + & + & + & $\mathrm{Y}$ & $\mathrm{V}$ \\
\hline \multicolumn{6}{|l|}{ BACILLARIOPHYCEAE } \\
\hline Asterionella formosa Hassall & + & + & + & $\mathrm{C}$ & VI \\
\hline Aulacoseira granulata (Ehrenberg) Simonsen & + & + & + & $\mathrm{P}$ & VI \\
\hline Coconeis sp. & & & + & & \\
\hline Cymatopleura solea (Brébisson) W.Smith & + & + & + & & \\
\hline Cymbella spp. & + & + & + & & \\
\hline Diatoma sp. & & & + & & \\
\hline Epithemia sorex Kützing & + & + & & & \\
\hline Fragilaria crotonensis Kitton & + & + & + & & \\
\hline Fragilaria spp. & + & + & + & & \\
\hline
\end{tabular}


Fitoplancton de lagos urbanos eutróficos, Chile central: AlmanZA, V. ET AL.

\begin{tabular}{|c|c|c|c|c|c|}
\hline TAXON & LG & LM & LTP & FGs & MBFGs \\
\hline Fragilaria ulna (Nitzsch) Lange-Bertalot & + & & + & & \\
\hline Gomphonema acuminatum Ehrenberg & & & + & & \\
\hline Gomphonema sp. & + & + & + & & \\
\hline Gonphoneis sp. & + & & & & \\
\hline Melosira varians C.Agardh & + & + & + & $\mathrm{Tb}$ & VI \\
\hline Navicula spp. & + & + & + & & \\
\hline Nitzschia acicularis (Kützing) W.Smith sp1 & & & + & & \\
\hline Nitzschia dissipata (Kützing) Rabenhorst sp2 & + & + & + & & \\
\hline Nitzschia sigmoidea (Nitzsch) W.Smith & & & + & & \\
\hline Pinnularia similis Krasske & + & + & + & & \\
\hline Pleurosigma sp. & + & & + & & \\
\hline Surirella sp. & & & + & & \\
\hline Tabelaria sp. & & & + & & \\
\hline Ulnaria ulna (Nitzsch) Compère & + & + & + & & \\
\hline \multicolumn{6}{|l|}{ CYANOPHYCEAE } \\
\hline Aphanocapsa delicatissima W.West \& G.S.West & + & & & & \\
\hline Aphanocapsa elachista W.West \& G.S.West & + & + & & & \\
\hline Aphanocapsa incerta (Lemmermann) G.Cronberg \& Komárek & + & & & & \\
\hline Aphanocapsa koordesii Strøm & & + & & & \\
\hline Aphanothece minutissima (West) J.Komárková-Legnerová \& G.Cronberg & & + & + & & \\
\hline Aphanothece smithii J.Komárková-Legnerová \& G.Cronberg & & + & & $\mathrm{K}$ & \\
\hline Dolichospermum sp. & & + & & $\mathrm{Sn}$ & III \\
\hline Limnococcus sp. & & & + & & \\
\hline Limnothrix sp. & & + & + & & \\
\hline Merismopedia sp. & + & & + & & \\
\hline Microcystis aeruginosa (Kützing) Kützing & + & + & + & M & VII \\
\hline Microcystis wesenbergii (Komárek) Komárek ex Komárek & & + & & & \\
\hline Oscillatoria sp. & & + & + & & \\
\hline Phormidium Kützing ex Gomont & + & + & + & & \\
\hline Planktothrix agardhii (Gomont) Anagnostidis \& Komárek & & & + & & \\
\hline Pseudanabaena catenata Lauterborn & & + & & & \\
\hline Pseudanabaena mucicola (Naumann \& Huber-Pestalozzi) Schwabe & + & & + & & \\
\hline Snowella sp. & & & + & & \\
\hline Spirulina sp. & & + & & & \\
\hline \multicolumn{6}{|l|}{ DINOPHYCEAE } \\
\hline Ceratium furcoides (Levander) Langhans & & + & + & Lo & $\mathrm{V}$ \\
\hline Ceratium hirundinella (O.F.Müller) Dujardin & + & & + & Lo & $\mathrm{V}$ \\
\hline Gymnodinium sp. & & + & & & \\
\hline Peridiniopsis elpatiewskyi (Ostenfeld) Bourrelly & & + & & & \\
\hline Peridinium sp. 1 & & + & + & & \\
\hline Peridinium sp. 2 & & & + & & \\
\hline Peridinium willei Huitfeldt-Kaas & + & & & Lo & $\mathrm{V}$ \\
\hline \multicolumn{6}{|l|}{ EUGLENOPHYCEAE } \\
\hline Euglena sanguinea Ehrenberg & + & + & + & Wi & $\mathrm{V}$ \\
\hline Lepocinclis sp. & & & + & & \\
\hline Phacus sp. & + & + & + & $\mathrm{Y}$ & $\mathrm{V}$ \\
\hline Trachelomonas sp. & + & + & + & & \\
\hline \multicolumn{6}{|l|}{ XANTHOPHYCEAE } \\
\hline Tribonema sp. & & + & + & $\mathrm{T}$ & \\
\hline
\end{tabular}



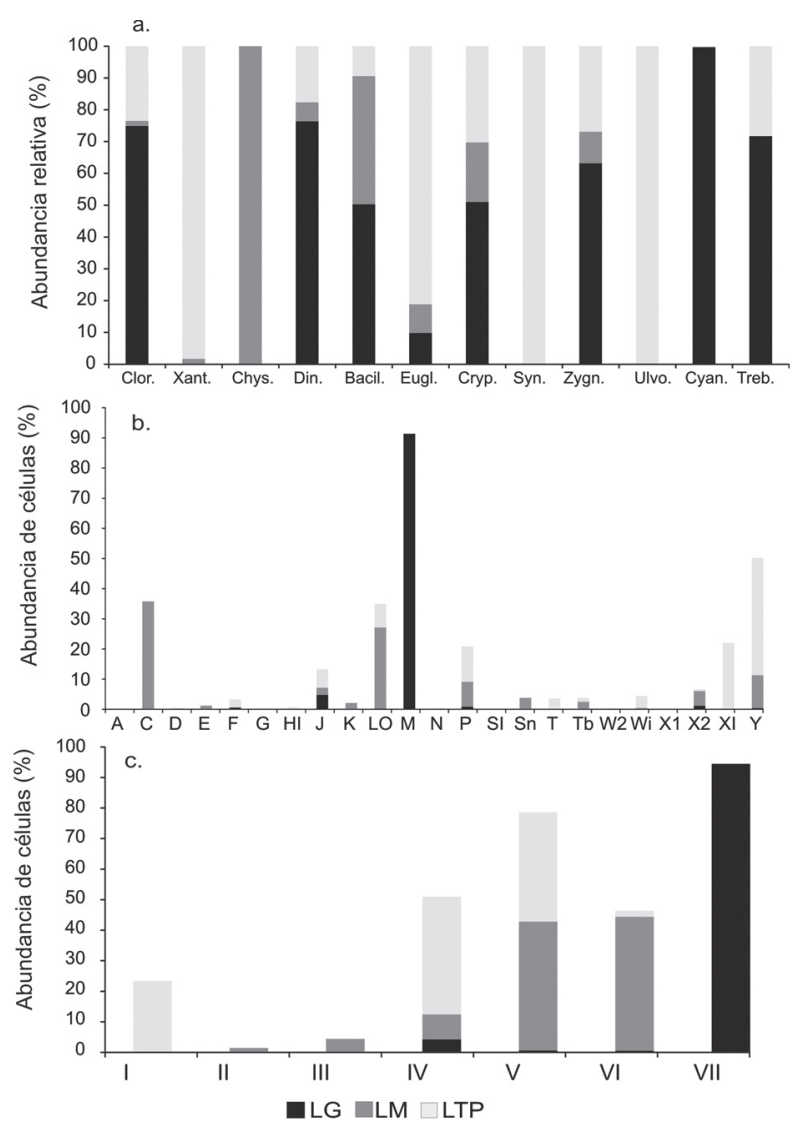

FIgURA 2. a. Abundancia relativa de microalgas identificadas en cada uno de los lagos: Clor. (Chlorophyceae), Xant. (Xanthophyceae), Chrys. (Chrysophyceae), Din. (Dinophyceae), Bacil. (Bacillariaceae), Eugl. (Euglenophyceae), Cryp. (Cryptophyceae), Syn. (Synurophyceae), Zygn. (Zygnematophyceae), Ulvo. (Ulvophyceae), Cyan.(Cyanophyceae)y Treb.(Trebouxiophyceae). b. Grupos funcionales FGs, c. MBFGs.

FIGURE 2. a. Relative abundance of algae in the lakes; Clor. (Chlorophyceae), Xant. (Xanthophyceae), Chrys. (Chrysophyceae), Din. (Dinophyceae), Bacil. (Bacillariaceae), Eugl. (Euglenophyceae), Cryp. (Cryptophyceae), Syn. (Synurophyceae), Zygn. (Zygnematophyceae), Ulvo. (Ulvophyceae), Cyan. (Cyanophyceae) y Treb. (Trebouxiophyceae). b. FGs functional groups, c. MBFGs.

Variaciones en la estructura de la comunidad fitoplanctónica de cada lago fueron significativas entre las estaciones del año (ANOSIM $\mathrm{R}_{\text {global }}=0,07 ; \mathrm{p}<0,05$ ), dadas principalmente por los aumentos de abundancia de algunos taxones dominantes como $M$. aeruginosa, $C$. furcoides y $A$. formosa. La contribución de los taxones en determinar las diferencias en la estructura de la comunidad de cada lago se presentan en la Tabla III.

En LG la comunidad fue dominada por cianobacterias y algas verdes (Fig. 4a). En total se registraron 80 taxones de ocho clases y la mayor abundancia y diversidad H'(loge) (3.0); sin embargo, se observó una menor cantidad de grupos funcionales (5) con respecto a los otros dos lagos, los FGs dominantes en el lago fueron $\mathrm{M} \rightarrow \mathrm{J} \rightarrow \mathrm{X} 2 \rightarrow \mathrm{F}$. La cianobacteria $M$. aeruginosa (Codón $\mathrm{M}$ ) determinó las diferencias en la composición de la comunidad con respecto a LM y LTP. Esta especie generó una floración acumulativa en verano de 2013 y varias floraciones dispersivas en otras estaciones del año (verano de 2012 y 2014, invierno y primavera de 2013), las que fueron acompañadas por taxones del Codón J, principalmente Desmodesmus communis (E.Hegewald) E.Hegewald, Coelastrum microporum Nägeli, Acutodesmus acuminatus (Lagerheim) P.M.Tsarenko, Monactinus simplex (Meyen) Corda y Pediastrum duplex Meyen. Estas microalgas generaron aumentos de abundancia durante la primavera de 2012, el otoño de 2013 y el verano de 2014 (Fig. 3b). Los MBFGs más abundantes fueron VII (grandes colonias mucilaginosas como $M$. aeruginosa), IV (organismos de tamaño medio que carecen de características especializadas p.ej. especies de Monactinus, Pediastrum, Desmodesmus, Monoraphidium), VI (organismos no flagelados con exoesqueletos silíceos, como $A$. formosa).

En LM se registraron 63 taxones, de 11 clases diferentes. Esta comunidad estuvo dominada por diatomeas y dinoflagelados (Fig. 4c); presentó una diversidad de 2,5 y 10 diferentes FGs (principalmente $\mathrm{C} \rightarrow \mathrm{L}_{\mathrm{o}} \rightarrow \mathrm{Y} \rightarrow \mathrm{P}$ ). Se identificaron floraciones del dinoflagelado $C$. furcoides (Codón $\mathrm{L}_{\mathrm{o}}$ ) durante el otoño de dos años consecutivos (2012 y 2013) y de A. formosa (Codón C) en el verano y finales del invierno de los tres años estudiados; estas especies constituyeron la principal diferencia con respecto a los otros lagos (Fig. 4d). Aumentos de abundancia de las cianobacterias Aphanothece sp. y Dolichospermum sp. también fueron documentados en verano de 2012 y primavera de 2014, respectivamente (Fig. 4c). El grupo V (flagelados unicelulares de tamaño mediano a grande, como C. furcoides) (MBFGs) fue dominante en este lago, junto con los grupos morfológicos VI y IV, al igual que en LG y LTP.

En LTP se observaron representantes de las nueve clases registradas y de 11 diferentes FGs (principalmente $\mathrm{Y} \rightarrow \mathrm{X} 1$ $\rightarrow \mathrm{P} \rightarrow$ Lo). La comunidad estuvo dominada por las algas verdes (Chlorophyceae y Trebouxiophyceae), euglenofíceas (Euglenophyceae) y criptofíceas (Cryptophyceae). En este lago se registró la mayor riqueza de taxones (84), la que fue significativamente diferente con respecto a los otros lagos $(\mathrm{F}$ $=23,8$; g.1. 2, $6 ; \mathrm{p}<0,05)$, pero no varió entre las estaciones del año $(F=1,9 ;$ g.l. 2, 6; $\mathrm{p}<0,05)$ y una diversidad $H^{\prime}$ (loge) promedio de 2,4 significativamente diferente entre lagos y épocas $(F=2,6$; g.l. 1,$6 ; p<0,05)$. Se diferenciaron dos comunidades para cada una de las cubetas que conforman el lago, en la cubeta de menor profundidad el alga más abundante fue Ankyra judayi (G.M.Smith) Fott (Codón X1) que codominó con Phacus sp. (Codón Y). Sphaerocystis schroeteri Chodat, Staurastrum pingue Teiling (Codón P) y Cryptomonas ovata Ehrenberg (Codón Y, grupo V) dominaron en la cubeta de mayor profundidad. Aumentos de 
abundancia de las algas verdes fueron observados durante largos periodos en los años estudiados (desde octubre de 2012 al verano de 2013), y posteriormente en primavera de 2013 y 2014. Las euglenofíceas fueron abundantes en verano de 2012 y otoño e invierno de 2013 (Fig. 4e). En verano de 2014, se generó una floración de la dinofícea $C$. furcoides (Fig. 4f).

\section{CARACTERÍSTICAS AMBIENTALES}

Los resultados del CCA mostraron que la temperatura $(\mathrm{T})$, los nitritos $\left(\mathrm{NO}_{2}\right)$, la relación $\mathrm{N} / \mathrm{P}(\mathrm{N} / \mathrm{P})$, la conductividad y transparencia fueron los principales factores que influyeron en el desarrollo de las comunidades en todos los lagos. Diferencias de estos parámetros en cada lago generan una variación en la estructura de la comunidad y el dominio de uno u otro taxón (Fig. 5). En LG se presentaron los mayores valores promedio de NT y PT y la mayor abundancia de fitoplancton (clorofila-a) que se desarrolló a un $\mathrm{pH}$ básico (el mayor en comparación a los otros lagos).

En LTP, la conductividad y la transparencia del agua fueron las más altas durante todo el periodo estudiado, al igual que las concentraciones de nitrato $\left(\mathrm{NO}_{3}\right)$ y amonio $\left(\mathrm{NH}_{4}\right)$ (Tabla IV). Estas características permitieron el aumento de la abundancia de uno a varios taxones de algas verdes al mismo tiempo, sin llegar a generar floraciones. En LM la concentración de $\mathrm{NT}_{\text {y }} \mathrm{NO}_{3}$ fue menor en comparación con los otros lagos, y el pH tendió a ser más neutro. Estas condiciones favorecieron el desarrollo de floraciones del dinoflagelado $C$. furcoides y de la diatomea $A$. formosa. En este lago la concentración de fosfato $\left(\mathrm{PO}_{4}\right)$ fue mayor con respecto a los demás lagos (Tabla IV).
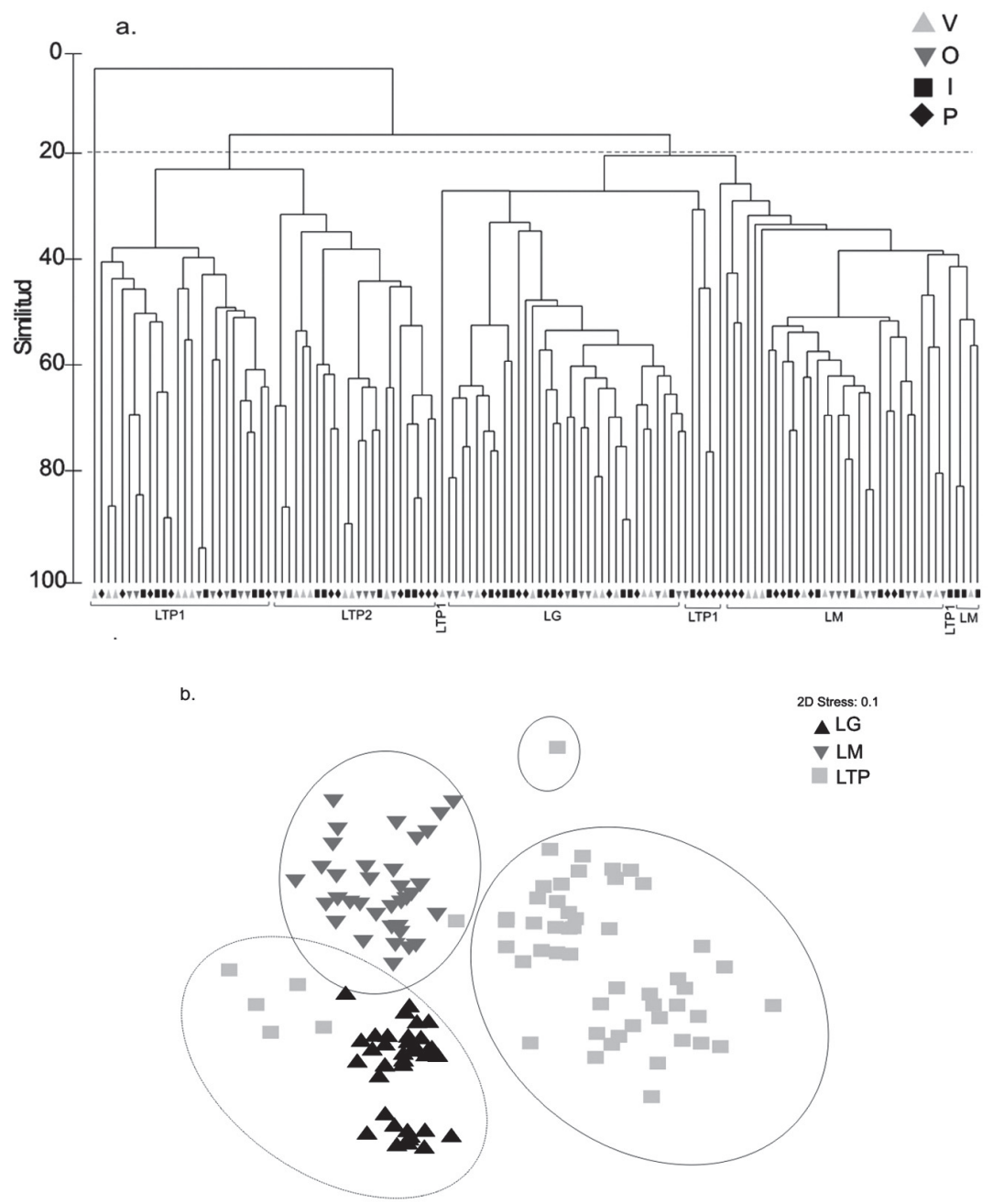

Figura 3. a. Análisis de cluster y b. ordenamiento multidimensional no métrico (MDS) de abundancia promedio de microalgas (transformación $\log \mathrm{x}+1$; Índice de similitud de Bray-Curtis) en cada lago y estación del año. $\mathrm{V}=$ verano, $\mathrm{O}=$ otoño, $\mathrm{I}=$ invierno, $\mathrm{P}=$ primavera. LTP= Laguna Las Tres Pascualas, LTP1 (estación 1), LTP2 (estación 2), LG=Lo Galindo, LM=Lo Méndez.

Figure 3. a. Cluster analysis and $\mathrm{b}$. Non-metric multidimensional scaling of microalgae taxa (MDS) (transformation log $\mathrm{x}+1$; Index BrayCurtis similarity) in each lake and season. $\mathrm{V}=$ summer, $\mathrm{O}=$ autumn $\mathrm{I}=$ winter, $\mathrm{P}=$ spring. LTP1 (station 1), LTP2 (station 2), LG=Lo Galindo, LM=Lo Méndez. 
TABLA III. Especies características que definen la disimilitud entre los lagos urbanos. Resultados obtenidos con el análisis SIMPER de PRIMER. AP = abundancia promedio; $\mathrm{DIP}=$ disimilitud promedio; $\mathrm{DS}=$ desviación estándar; $\mathrm{CTB}=$ contribución porcentual; $\mathrm{ACM}=$ porcentaje acumulativo; Dis $=$ disimilitud.

TABLE III. Characteristic species that define the dissimilitude between the lakes. Results obtained with the analysis SIMPER PRIMER. $\mathrm{AP}=$ average abundance; DIP = average dissimilarity; $\mathrm{SD}=$ standard deviation; $\mathrm{CTB}=$ percentage contribution; $\mathrm{ACM}=$ cumulative percentage; Dis = dissimilarity.

\begin{tabular}{|c|c|c|c|c|c|c|c|}
\hline LaGO & ESPECIE & $\begin{array}{c}\text { GRUPO } 1 \\
\text { AP }\end{array}$ & $\begin{array}{c}\text { GRUPO } 2 \\
\text { AP }\end{array}$ & DIP & Dis/SD & $\mathrm{CTB}$ & $\mathrm{ACM}$ \\
\hline \multirow{3}{*}{$\begin{array}{l}\text { LG \& LM } \\
(81.52 \%)\end{array}$} & Microcystis aeruginosa & 12.25 & 0 & 6.02 & 2.74 & 7.39 & 7.39 \\
\hline & Ceratium furcoides & 0 & 6.42 & 3.10 & 1.90 & 3.80 & 11.18 \\
\hline & Desmodesmus communis & 8.55 & 2.60 & 2.95 & 1.79 & 3.62 & 14.80 \\
\hline \multirow{3}{*}{$\begin{array}{l}\text { LG \& LTP } \\
(84.91 \%)\end{array}$} & Microcystis aeruginosa & 12.25 & 0.58 & 6.02 & 2.54 & 7.09 & 7.09 \\
\hline & Desmodesmus communis & 8.55 & 1.27 & 3.71 & 2.45 & 4.37 & 11.46 \\
\hline & Coelastrum microporum & 7.03 & 0.77 & 3.12 & 2.09 & 3.68 & 15.14 \\
\hline \multirow{3}{*}{$\begin{array}{l}\text { LM \& LTP } \\
(83.72 \%)\end{array}$} & Ceratium furcoides & 6.42 & 0 & 4.91 & 1.88 & 5.87 & 5.87 \\
\hline & Asterionella formosa & 6.22 & 0.44 & 4.53 & 1.72 & 5.41 & 11.28 \\
\hline & Cryptomonas ovata & 5.87 & 3.16 & 3.18 & 1.33 & 3.79 & 15.07 \\
\hline
\end{tabular}
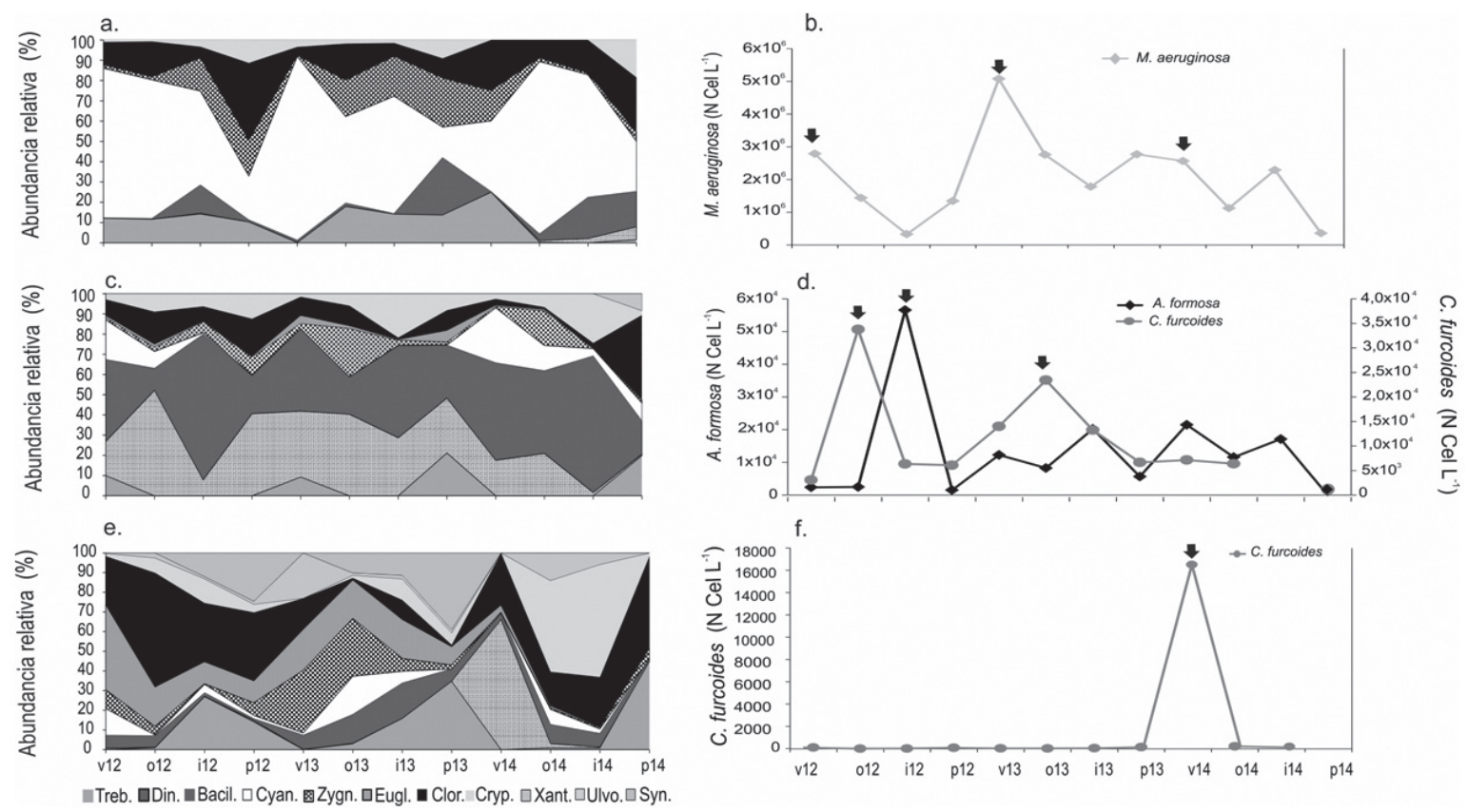

FIgURA 4. Variación estacional de la abundancia relativa de los diferentes grupos de fitoplancton en los lagos someros durante el periodo de estudio, a. Lo Galindo, c. Lo Méndez, e. Las Tres Pascualas. Variación estacional de la abundancia de los taxones más abundantes y/o formadores de floraciones en los lagos urbanos, b. Lo Galindo, d. Lo Méndez, f. Las Tres Pascualas. Clor. (Chlorophyceae), Xant. (Xanthophyceae), Chrys. (Chrysophyceae), Din. (Dinophyceae), Bacil. (Bacillariaceae), Eugl. (Euglenophyceae), Cryp. (Cryptophyceae), Syn. (Synurophyceae), Zygn. (Zygnematophyceae), Ulvo. (Ulvophyceae), Cyan. (Cyanophyceae) y Treb. (Trebouxiophyceae). $v=$ verano, $\mathrm{o}=$ otoño, $\mathrm{i}=$ invierno, $\mathrm{p}=$ primavera. 12 = año $2012,13=$ año $2013,14=$ año 2014.

FIGURE 4. Seasonal variation in the relative abundance of the different phytoplankton groups in shallow lakes. a. Lo Galindo, c. Lo Méndez, e. Las Tres Pascualas. Seasonal variation most abundant taxa and / or forming blooms in urban lakes, b. Lo Galindo, d. Lo Méndez, f. Tres Pascualas. Clor. (Chlorophyceae), Xant. (Xanthophyceae), Chrys. (Chrysophyceae), Din. (Dinophyceae), Bacil. (Bacillariaceae), Eugl. (Euglenophyceae), Cryp. (Cryptophyceae), Syn. (Synurophyceae), Zygn. (Zygnematophyceae), Ulvo. (Ulvophyceae), Cyan. (Cyanophyceae) y Treb. (Trebouxiophyceae) $\mathrm{v}=$ summer, $\mathrm{o}=$ autumn, $\mathrm{i}=$ winter, $\mathrm{p}=$ spring. $12=$ year 2012, year $13=2013,14=$ year 2014 . 


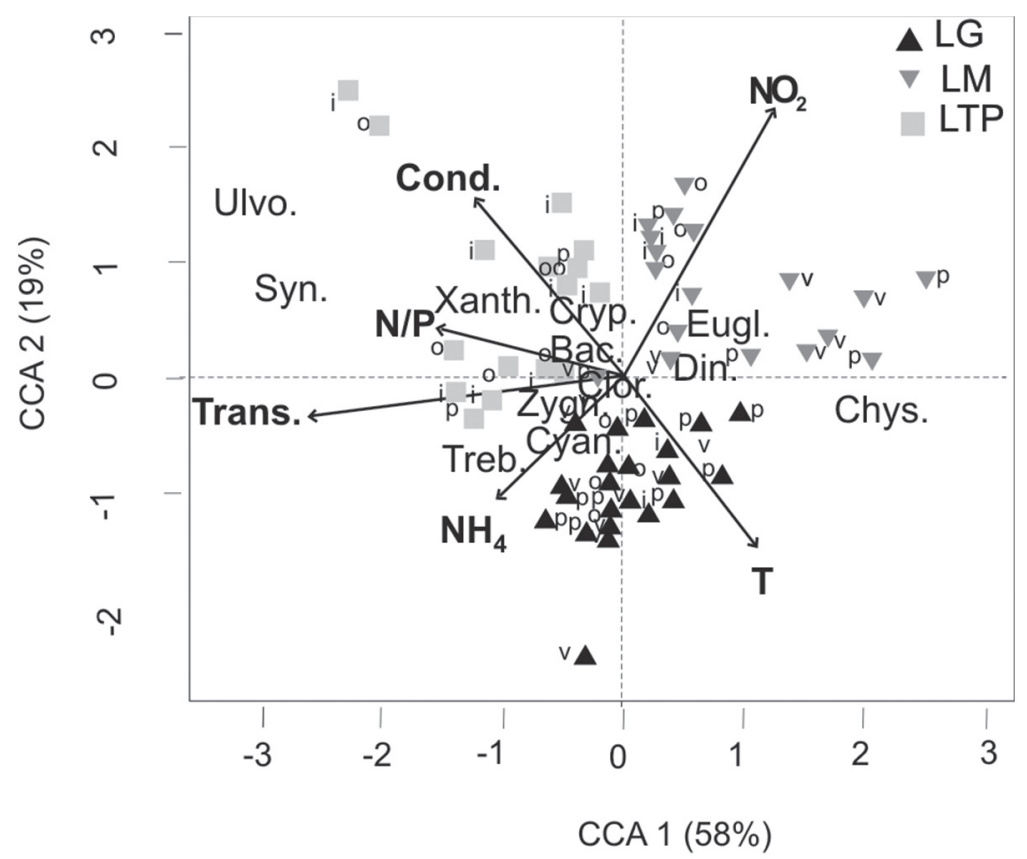

Figura 5. Análisis de correspondencia canónica (CCA), abundancia (cel L L-1) de grupos de fitoplancton en cada lago somero y estación del año. Cloro (Chlorophyceae), Cyano (Cyanobacteria), Bacil. (Bacillariaceae), Dino (Dinophyceae), Chryso (Chrysophyceae), Eugleno. (Euglenophyceae), Crypto (Cryptophyceae), Synu (Synurophyceae) y Xanto (Xanthophyceae). V=verano, O=otoño, I=invierno, $\mathrm{P}=$ primavera.

FiguRE 5. Canonical correspondence analysis (CCA), phytoplankton groups abundance (cel $\mathrm{L}^{-1}$ ) in shallow lake each season. Cloro (Chlorophyceae), Cyano (Cyanobacteria), Bacil. (Bacillariaceae), Dino (Dinophyceae), Chryso (Chrysophyceae), Eugleno. (Euglenophyceae), Crypto (Cryptophyceae), Synu (Synurophyceae) and Xanto (Xanthophyceae). V=summer, O=autumn, I=winter, $\mathrm{P}=$ spring.

TABLA IV. Parámetros físicos y químicos en los lagos urbanos someros durante el periodo estudiado. Promedio (Mín.-Máx).

TABLE IV. Physical and chemical parameters in urban shallow lakes during the study. Average (Min-Max).

\begin{tabular}{lccc}
\hline LAGO & LG & LM & LTP \\
\hline $\mathrm{NT}\left(\mathrm{mg} \mathrm{L}^{-1}\right)$ & $2.11(<0.01-6.00)$ & $1.41(0.02-5.22)$ & $1.66(0.45-2.92)$ \\
$\mathrm{NO}_{3}\left(\mathrm{mg} \mathrm{L}^{-1}\right)$ & $1.28(0.01-4.69)$ & $0.73(0.05-4.30)$ & $1.71(0.01-8.54)$ \\
$\mathrm{NO}_{2}\left(\mathrm{mg} \mathrm{L}^{-1}\right)$ & $0.10(<0.02-1.00)$ & $0.02(<0.02-0.08)$ & $0.04(<0.02-0.18)$ \\
$\mathrm{NH}_{4}\left(\mathrm{mg} \mathrm{L}^{-1}\right)$ & $0.10(<0.02-0.58)$ & $0.21(<0.02-1.82)$ & $0.35(<0.02-1.33)$ \\
$\mathrm{PT}\left(\mathrm{mg} \mathrm{L}^{-1}\right)$ & $0.35(0.09-2.00)$ & $0.08(0.03-0.33)$ & $0.09(0.03-0.33)$ \\
$\mathrm{PO}_{4}\left(\mathrm{mg} \mathrm{L}^{-1}\right)$ & $0.1(<0.04-0.80)$ & $0.20(<0.04-1.0)$ & $<0.04$ \\
Temp. $\left({ }^{\circ} \mathrm{C}\right)$ & $19.26(8.71-26.30)$ & $19.04(9.13-28.50)$ & $17.20(9.24-24.00)$ \\
Relación NT:PT & 6.02 & 17.62 & 18.44 \\
Transparencia $(\mathrm{m})$ & $0.34(0.12-0.60)$ & $1.12(0.20-2.90)$ & $1.49(0.80-3.50)$ \\
Cond. $\left.(\mu \mathrm{S} \mathrm{cm})^{-1}\right)$ & $259.75(156.00-418.00)$ & $258.08(113.50-472.00)$ & $329.09(232.00-483.00)$ \\
pH & $8.29(6.44-10.07)$ & $7.73(6.34-8.70)$ & $8.18(5.80-9.43)$ \\
OD $\left(\mathrm{mg} \mathrm{L}^{-1}\right)$ & $8.34(5.52-13.60)$ & $8.02(3.10-13.66)$ & $8.02(2.04-15.10)$ \\
Clorofila-a $(\mu \mathrm{g} \mathrm{L})$ & $85.15(15.07-330.41)$ & $59.29(4.70-351.59)$ & $25.30(1.67-138.52)$ \\
\hline
\end{tabular}




\section{DISCUSION}

La comunidad de fitoplancton de los tres lagos urbanos estudiados es típica de ambientes meso- a eutróficos según la clasificación taxonómica, FGs y MBFGs. Estos hábitats soportan una elevada densidad de microalgas y poseen una alta productividad con pocas especies dominantes como M. aeruginosa (Codón M, grupo VII), C. furcoides (Codón $\mathrm{L}_{\mathrm{o}}$, grupo V), A. formosa (Codón C, grupo VI), C. ovata (Codón $\mathrm{Y}$, grupo $\mathrm{V}$ ) y un grupo de algas conformado por D. opoliensis, A. acuminatus, M. simplex, P. duplex, C. microporum, S. schroeteri y S. gracile (Codón J, grupo IV).

Aunque los lagos estudiados se encuentran en la misma zona geográfica y sometidos a las mismas condiciones ambientales de luz, precipitaciones y temperatura ambiental, la comunidad fitoplanctónica responde de forma diferente a las características fisicoquímicas propias de cada lago, lo que se refleja en una composición de grupos taxonómicos diferentes; en LG la comunidad está dominada por Cyanophyceae y Chlorophyceae, en LM por Dinophyceae y Bacillariaceae y en LTP las Chlorophyceae (A. judayi, $S$. schroeteri), Zygnematophyceae (S. pingue), Cryptophyceae (C. ovata) y Euglenophyceae constituyen los principales grupos de la comunidad fitoplanctónica. Las características propias de cada lago determinan diferencias principalmente en la transparencia y conductividad de la columna de agua, así como en la concentración de nutrientes (relación NT/ PT), que finalmente condicionan el desarrollo de diferentes comunidades de fitoplancton.

En cada lago la estructura de la comunidad presentó variaciones estacionales, en concordancia con lo reportado por Parra et al. (1981) para estos sistemas. Las floraciones de dinoflagelados (C. furcoides en otoño), diatomeas ( $A$. formosa en verano e invierno) y aumentos de algas verdes (M. simplex, P. duplex, D. opoliensis, A. acuminatus y $C$. microporum en primavera y verano) variaron estacionalmente en todos los lagos estudiados, a diferencia de las floraciones de $M$. aeruginosa que se presentaron durante todo el año (acumulativas en verano de 2013 y dispersivas en todas las estaciones).

En particular el lago LG, con altas concentraciones de NT y PT, presentó una mayor abundancia y diversidad de taxones, así como densas floraciones de la cianobacteria no fijadora de nitrógeno, M. aeruginosa (grupo VII). La presencia de esta especie en MBFG se relaciona con características eutróficas (Kruk \& Segura 2012). Cuando ambos nutrientes, tanto el NT y PT, existen en una alta concentración se genera un rápido incremento de la abundancia de microalgas (Schindler 2015). Cuando la razón N:P es menor (p.ej. <7) sugiere una posible limitación relativa de nitrógeno y favorece la formación de floraciones de cianobacterias (Bicudo et al. 1999, Pael et al. 2001, Schindler et al. 2008). Por el contrario, una mayor relación N:P (p.ej. > 16) determina un aumento de algas verdes y diatomeas sugiriendo, en este caso, una limitación por fósforo en el ambiente (Carpenter et al. 1996, Bicudo et al. 1999), situación que se reflejaría en los lagos urbanos LTP y LM.

Al aumentar la concentración de nutrientes hacia condiciones hipereutróficas, las cianobacterias alternan su dominancia con las algas verdes (Reynolds 2006). Durante los periodos de mayor disponibilidad de nitratos se observaron colonias macroscópicas de M. aeruginosa disgregadas por toda la columna de agua sin llegar a conformar una densa capa (floración dispersiva). Estas floraciones, se presentaron junto con una alta abundancia de varias especies de algas verdes como $P$. boryanum, D. communis, D. opoliensis, $A$. acuminatus, $M$. simplex, C. microporum y $S$. schroeteri. En lagos eutróficos someros, se ha reportado principalmente a $P$. boryanum y $D$. communis, como acompañantes comunes de las floraciones dispersivas de $M$. aeruginosa y típicas habitantes de lagos eutróficos (Chaudhary \& Meena 2007). En entornos ricos en nitratos, esta especie de Microcystis queda fuera de competencia con otro tipo de fitoplancton debido a su baja tasa de asimilación, en esta situación las algas verdes de rápido crecimiento pueden dominar y reemplazar a las especies de crecimiento lento como las cianobacterias (Jacoby et al. 2000). La coexistencia entre floraciones de M. aeruginosa (Codón M y grupo VII) y del grupo de algas verdes del Codón J en LG, implican una respuesta común a las mismas condiciones ambientales. En condiciones de altas temperaturas (incremento anual 25 ${ }^{\circ} \mathrm{C}$ ), máxima concentración de fosfatos y nitrógeno total, así como disminución de la velocidad del viento, las colonias de $M$. aeruginosa se agruparon y formaron capas de varios centímetros de espesor en la superficie del agua formando floraciones acumulativas y/o "scums" (Almanza et al. 2016b).

En LM se registró la mayor concentración de $\mathrm{PO}_{4}$ con respecto a los otros dos lagos. El dinoflagelado $(C$. furcoides) y la diatomea ( $A$. formosa) representaron más del $70 \%$ de la abundancia total del fitoplancton, las que alternan su dominancia generando floraciones en diferentes épocas del año: $A$. formosa (C-codón C) en verano e invierno y $C$. furcoides (Codón $\mathrm{L}_{\mathrm{o}}$ ) en otoño. Según Krivtsov et al. (2000), las floraciones de $A$. formosa decrecen rápidamente por la disponibilidad de la concentración de silicio en el ambiente, constituyendo el principal factor que incide en la ocurrencia de floraciones de diatomeas en los lagos. Las diatomeas son más eficientes que los dinoflagelados en bajas intensidades de luz (Ryther 1956), lo que podría determinar en gran medida la secuencia de floraciones de estos dos grupos en LM entre las estaciones del año.

En LTP la mayor concentración promedio de $\mathrm{NH}_{4}$ y $\mathrm{NO}_{3}$, la conductividad y la morfología arriñonada con dos cubetas del lago, determinaron una comunidad con mayor riqueza de taxones, así como de grupos funcionales con respecto a los otros lagos, estableciendo una comunidad fitoplanctónica heterogénea espacialmente. En este 
lago predominaron microalgas oportunistas, de rápido crecimiento y reproducción, generalmente de tamaños pequeños y alta relación superficie/volumen, denominadas C-estrategas (como A. judayi y C. ovata) (Reynolds 2006) y representantes del Codón Y y grupo XI, típicos habitantes de lagos someros y ambientes eutróficos (Padisák et al. 2009). Una sola floración de C. furcoides fue evidente en el lago durante el periodo de estudio, también fueron registrados aumentos de abundancia de C. ovata, A. judayi, S. pingue y euglenofíceas (Phacus sp.).

Durante el desarrollo de las floraciones, de C. furcoides y el "scums" de $M$. aeruginosa, se registró un aumento en la concentración de clorofila-a y oxígeno disuelto, persistencia de $\mathrm{pH}$ básico $(>8)$ y una disminución en la concentración de formas inorgánicas de nutrientes (principalmente fosfatos), sugiriendo una reducción o agotamiento por su incorporación en la biomasa algal (Springer et al. 2005). La rápida asimilación de los nutrientes impulsa el reciclaje de los mismos de una manera que desplaza competitivamente a otros grupos del fitoplancton (Elser 1999).

Es relevante el hecho de la formación de floraciones a bajas temperaturas como las de otoño e invierno en los tres lagos estudiados. En la zona templada se reconocía el hecho, al menos en lagos de América del Norte y Europa, de la exclusión de especies formadoras de floraciones en invierno por las bajas temperaturas (Reynolds 2006). Sin embargo, los resultados muestran floraciones durante las estaciones de otoño (p. ej. entre $12-19{ }^{\circ} \mathrm{C}$ de $C$. furcoides) e invierno (p. ej. a $10^{\circ} \mathrm{C}$ una floración dispersiva de $M$. aeruginosa y $13{ }^{\circ} \mathrm{C} \mathrm{A}$. formosa) a temperaturas inferiores a las reportadas en proliferaciones de otros lagos tropicales y del desarrollo óptimo de las especies. Lo anterior estaría indicando que en condiciones de exceso de nutrientes estos lagos sostienen altas tasas de crecimiento tanto de algas eucariotas y/o procariotas a pesar de la oscilación en la temperatura del agua durante el ciclo anual. Un aumento de la temperatura en los meses de invierno significaría densas floraciones de fitoplancton durante todo el año.

El dinoflagelado $C$. furcoides ha sido reconocido como una especie invasora en sur América, que en poco tiempo ha aumentado su área de distribución (Calvacante et al. 2016). Meichtry et al. (2016) determinaron que la temperatura restringe el desarrollo de esta especie y que los entornos más susceptibles a la invasión serían aquellos con una temperatura media anual entre 18 y $22^{\circ} \mathrm{C}$, sugiriendo que los cuerpos de agua de Chile, no son susceptibles a la invasión. Los resultados de este estudio demuestran que hábitats con alta concentración de nutrientes como los registrados en LM y LTP, favorecen no solo el establecimiento del dinoflagelado sino también su proliferación.

La frecuencia y periodicidad de las floraciones de microalgas registrada en estos sistemas es un indicador del proceso de eutrofización progresiva (Almanza et al. 2016a) y del deterioro de la calidad del agua de los lagos urbanos de Concepción, lo que puede conllevar a consecuencias negativas para el uso de estos cuerpos de agua, por la disminución del valor estético, además del posible riesgo para la salud humana y animal por la presencia de cianotoxinas (Almanza et al. 2016b). El manejo de las floraciones en los lagos urbanos de Concepción debe ser tomado en consideración al momento de generar medidas de gestión y/o recuperación de los mismos.

Finalmente, las especies clasificadas tanto por FGs como por MBFGs reflejan las condiciones eutróficas de cada lago y representan similares resultados a los obtenidos por el análisis de CCA (producto de la clasificación taxonómica y de parámetros físicoquímicos tomados en los lagos estudiados). La aplicación de estos enfoques son una herramienta eficaz para explorar los efectos de los cambios ambientales sobre el fitoplancton independientemente de la ubicación geográfica y la composición específica (Kruk \& Segura 2012). La presencia de floraciones fitoplanctónicas en diferentes hábitats puede ser identificada principalmente por FGs, debido a que con el MBFGs la(s) especie(s) causante(s) de la floración puede(n) ser subestimada(s). En el caso de las floraciones tóxicas, este tipo de enfoque pierde resolución al intentar predecirlas (Kruk \& Segura 2012). Se recomienda utilizar el sistema de clasificación FGs para el monitoreo de los lagos de Concepción, junto con estudios complementarios para las floraciones de cianobacterias en Lo Galindo, si se pretende utilizar el agua para consumo y/o recreación.

\section{CONCLUSIONES}

En ambientes eutrofizados la comunidad de fitoplancton varía dependiendo de las características locales del hábitat, las que generan las condiciones apropiadas para el desarrollo y dominancia de ciertas especies de microalgas que pueden generar floraciones. La frecuencia y periodicidad de las floraciones de diferentes grupos de fitoplancton en los lagos urbanos de Concepción constituyen un indicador de la contaminación por nutrientes en estos cuerpos de agua (eutrofización cultural). En Lo Galindo se presentaron floraciones de cianobacterias y algas verdes, en Lo Méndez de dinoflagelados y diatomeas, mientras que en Las Tres Pascualas las algas verdes, criptofíceas y las euglenofíceas dominaron la comunidad, pero sin frecuentes eventos de floraciones. Los cambios en el sistema producidos por las floraciones influencian el uso actual y futuro de los lagos urbanos; su comprensión es necesaria para desarrollar estrategias de gestión de la calidad del agua.

\section{AGRADECIMIENTOS}

A las siguientes fuentes de financiamiento a los proyectos CRHIAM/Conicyt/Fondap 15130015; FONIS SA13I20211 y VRID No. 212.310.062.1.0. 


\section{BIBLIOGRAFÍA}

Almanza, V., R. Figueroa, O. Parra, X. Fernández, C. Baeza, J. YÁÑEZ \& R. URrutia. 2016a. Bases limnológicas para la gestión de los lagos urbanos de Concepción, Región del Biobío, Chile. Latin American Journal of Aquatic Research 44(2): 313-326.

Almanza, V., O. Parra, C.E. Bicudo, C. Baeza, J. Beltran, R. Figueroa \& R. URrutia. 2016b. Occurrence of toxic blooms of Microcystis aeruginosa in a central Chilean $\left(36^{\circ}\right.$ lat. S) urban lake. Revista Chilena de Historia Natural 89 8-20

American Public Health Association (APHA). 2012. Standard methods for examination of water and wastewater. American Public Health Association, Washington. 541 pp.

AndERSEN, R.A. 2007. Molecular systematics of the Chrysophyceae and Synurophyceae. In: J. Brodie \& J. Lewis (eds.) Unravelling the algae: the past, present, and future of algal systematics. The Systematic Association, Special Volume Ser. 75, pp. 285-314. CRC Press.

Bicudo, C.E.M., R.J.J. Ramírez, A. Tucci \& D.C. Bicudo. 1999. Dinâmica de populações fitoplanctônicas em ambientes eutrofizados: O Lago das Garças, São Paulo. En: R. Henry (ed.), Ecologia de reservatórios: estrutura, função e aspectos sociais. pp. 449-508. Botucatu, FUNDIBIO, FAPESP.

Bock, C., T. Pröschold \& L. Krienitz. 2011. Updating the genus Dictyosphaerium and description of Mucidosphaerium gen. nov. (Trebouxiophyceae) based on morphological and molecular data. Journal of Phycology 47: 638-652.

Calvacante, K.P., L. De Souza Cardoso, R. Sussella \& V. BECKER. 2016. Towards a comprehension of Ceratium (Dinophyceae) invasion in Brazilian freshwaters: autecology of $C$. furcoides in subtropical reservoirs. Hydrobiologia 771(1): 265-280.

Campos, V., S. Lisperguer, J. Weckesser, A. Vera \& D. Muñoz. 2005. Cyanobacteria and potential risks of toxicity in continental waters of Chile. Boletín Micológico 20: 73-81.

Carpenter, S.R., J.F. Kitchell, K.L. Cottingham, D.E. Schindler, D.L. Christensen, D.M. Post \& N. Voichick. 1996 Chlorophyll variability, nutrient input, and grazing: evidence from whole-lake experiments. Ecology 77: 725735 .

Carstensen, J., P. Henriksen \& A.S. Heiskanen. 2007. Summer algal blooms in shallow estuaries: definition, mechanisms, and link to eutrophication. Limnology and Oceanography 50(1): 370-384

Cerino, F. \& A. Zingone. 2007. Decrypting cryptomonads: a challenge for molecular taxonomy. In: J. Brodie \& J. Lewis (eds.), Unraveling the algae: the past, present, and future of algal systematics. The Systematic Association, Special Volume Ser 75, pp. 197-214. CRC Press.

Chaudhary, B.L. \& L. Meena. 2007. An environmental hazard - a case study of toxic bloom of Microcystis (Anacystis) spp. in Udaipur lakes Rajasthan (India). Journal of Herbal Medicine 1: 55-59.

Chol, B., M. Son, J.I. KIm \& W. ShIn. 2013. Taxonomy and phylogeny of genus Cryptomonas (Cryptophyceae, Cryptophyta) from Korea. Algae 28(4): 307-330.

Chorus, I. \& J. Bartram. 1999. Toxic cyanobacteria in water. A guide to their public health consequences monitoring and management. $1^{\mathrm{a}}$ ed., E \& FN Spon, London. 668 pp.

Clarke, K.R. \& R.M. WarWick. 2001. A further biodiversity index applicable to species lists: variation in taxonomic distinctness. Marine Ecology Progress Series 216: 265-278.

Davis, T.W., D.L. Berry, G.L. Boyer \& C.J. Gobler. 2009. The effects of temperature and nutrients on the growth and dynamics of toxic and nontoxic strains of Microcystis during cyanobacteria blooms. Harmful Algae 8: 715-725.

Elosegi, A. \& S. Sabater. 2009. Conceptos y técnicas en ecología fluvial. La biota de los ríos: los microorganismos autótrofos. En: A. Elosegui. \& S. Sabater (eds.), Conceptos y técnicas en ecología. pp. 26. Fundación BBVA, Bilbao, España.

ELSER, J.J. 1999. The pathway to noxious cyanobacteria blooms in lakes: the food web as the final turn. Freshwater Biology 42: $537-543$

Guiry, M.D. \& G.M. Guiry. 2016. AlgaeBase. World-wide electronic publication, National University of Ireland, Galway. http://www.algaebase.org

Hötzel, G. \& R. Croome. 1999. A phytoplankton methods manual for Australian freshwaters. LWRRDC Occasional Paper 22/99. Land and Water Resources Research and Development Corporation, Australia. 66 pp.

Imberger, J., T. Berman, R.R. Christian, E.B. Sherr, D.E. Whitney, L.R. Pomeroy, R.G. Wiegert \& W.J. Wiebe. 1983. The influence of water motion on the distribution and transport of materials in a salt marsh estuary. Limnology and Oceanography 28: 201-214.

Jacoby, J.M., D.C. Collier, E.B.F. Welch, J. Hardy \& M. CRAYTON. 2000. Environmental factors associated with a toxic bloom of Microcystis aeruginosa. Canadian Journal of Fisheries and Aquatic Sciences 57: 231-240.

KomÁreK, J. 2013. Cyanoprokaryota 3: Heterocytous Genera. In: B. Büdel, G. Gärtner, L. Krienitz \& M. Schagerl: Süßwasserflora von Mitteleuropa, Vol. 19/3, Spektrum Akademischer Verlag, 1130 pp.

KomáreK, J. \& K. Anagnostidis. 1999. Cyanoprocaryota - 1. Teil:Chroococcales.- In: H. Ettl, J. Gerloff, H. Heynig \& D. Mollenhauer (eds.). Süßwasserflora von Mitteleuropa, Band 19/1, Gustav Fischer Verlag. 548 pp.

KomÁreK, J. \& K. Anagnostidis. 2005. Cyanoprokaryota -2. Teil/ 2nd Part: Oscillatoriales. In: B. Büdel, L. Krienitz, G. Gärtner \& M. Schagerl (eds.), Süßwasserflora von Mitteleuropa 19/2, Elsevier/Spektrum, Heidelberg, 759 pp.

KrienitZ, L. \& C. Bock. 2012. Present state of the systematics of planktonic coccoid green algae of inland waters. Hydrobiologia 698: 295-326.

Krivtsov, V., E.G. Bellinger \& D.C. Sigee. 2000. Changes in the elemental composition of Asterionella formosa during the diatom spring bloom. Journal of Plankton Research 22: 169-184.

Kruk, C., V.L.M. Huszar, E.H.M. Peeters, S. Bonilla, L. Costa, M. Lurling, C.S. Reynolds \& M. Scheffer. 2010. A morphological classification capturing functional variation in phytoplankton. Freshwater Biology 55(3): 614-627.

Kruk, C. \& A.M. Segura. 2012. The habitat template of phytoplankton morphology-based functional groups. Hydrobiologia 698(1): 191-202.

Leliaert, F., D.R. Smith, H. Moreau, M.D. Herron, H. 
Fitoplancton de lagos urbanos eutróficos, Chile central: Almanza, V. ET AL.

Verbruggen, C.F. Delwiche \& O. De Clerck. 2012. Phylogeny and molecular evolution of the green algae. Critical Reviews in Plant Sciences 31: 1-46.

Moestrup, O. \& N. DaugBjerg. 2007. On dinoflagellate phylogeny and classification. In: J. Brodie \& J. Lewis (eds.), Unravelling the algae: the past, present, and future of algal systematics. The Systematic Association, Special Volume Ser. 75, pp. 215-230. CRC Press.

Marin, B., A. Palm, M. Klingberg \& M. Melkonian. 2003. Phylogeny and taxonomic revision of plastid-containing Euglenophytes based on SSU rDNA sequence comparisons and synapomorphic signatures in the SSU rRNA secundary structure. Protist 154: 99-145.

Meichtry, N., R.E. Vogler, M.J. Molina \& V.M. Llano. 2016. Potential distribution of the invasive freshwater dinoflagellate Ceratium furcoides (Levander) Langhans (Dinophyta) in South America. Journal of Phycology DOI: 10.1111/jpy.12382.

Padisák, J., L.O. Crossetti \& L. Naselli-Flores. 2009. Use and misuse in the application of the phytoplankton functional classification: a critical review with updates. Hydrobiologia 621: 1-19.

PAerL, H.W. 1988. Nuisance phytoplankton and inland waters blooms in coastal, estuarine. Water Resources Research 33: 823-847.

PAerL, H.W. 2014. Mitigating harmful cyanobacterial blooms in a human and climatically impacted world. Life 4: 988-1012.

Paerl, H.W., R.S. Fulton, P.H. Moisander \& J. Dyble. 2001. Harmful freshwater algal blooms, with an emphasis on Cyanobacteria. The Scientific World 1: 76-113.

Parra, O. 2009. Situación ambiental de los lagos de Concepción. Seminario Recuperemos los Lagos para los Habitantes de Concepción. Concepción. EULA-CHILE. 2 pp.

Parra, O., E. Ugarte \& V. Dellarossa. 1981. Periodicidad estacional y asociaciones en el fitoplancton de tres cuerpos lénticos en la región de Concepción, Chile. Gayana, Botánica 36: 1-35.

Parra, O., M. González, V. Dellarossa, P. Rivera \& M. Orellana. 1982-1983. Manual Taxonómico del Fitoplancton de Aguas Continentales; con especial referencia al fitoplancton de Chile. Editorial de la Universidad de Concepción, Concepción. Vol. 1, 70 pp., Vol. 2, 82 pp., Vol. 3, 99 pp., Vol. 4, 97 pp. y Vol. 5, 353 pp.

Parra, O., D. Avilés, J. Becerra, V. Dellarossa \& R. Montoya. 1986. Primer registro de floración de algas verde - azules en Chile: Informe preliminar. Gayana Botánica 43(1-4): 15-17.

Peretyatko, A., S. Teissier, J.J. Symoens \& L. Triest. 2007. Phytoplankton biomass and environmental factors over a gradient of clear to turbid peri-urban ponds. Aquatic Conservation: Marine and Freshwater Ecosystems 17: 584-601.

REynoldS, C.S. 2006. The ecology of freshwater phytoplankton. Cambridge University Press, Cambridge. 535 pp.

Reynolds, C.S., H. Vera, C. Kruk, L. Naselli-Flores \& S. Melo. 2002. Towards a functional classification of the freshwater phytoplankton. Journal of Plankton Research 24(5): 417-428.

Robarts, R.S. \& T. Zohary. 1987. Temperature effects on photosynthetic capacity, respiration and growth rates of bloom forming cyanobacteria. New Zealand Journal of Marine and Freshwater Research 21: 391-399.

Rumrich, U., H. Lange-Bertalot \& M. Rumrich. 2000. Diatomeen der Anden: Von Venezuela bis Patagonien/Feuerland und zwei weitere Beiträge. Iconographia Diatomológica. Volume 9. Koeltz Scientific Books, Koenigstein, Deutschland. $673 \mathrm{pp}$

Ryther, J.H. 1956. Photosynthesis in the ocean as a function of light intensity. Limnology and Oceanography 1: 61-70.

SchindLER, D.W. 2015. The dilemma of controlling cultural eutrophication of lakes. Proceedings of the Royal Society 5: 1-12.

Schindler, D.W., R.E. Hecky, D.L. Findlay, M.P. Stainton, B.R. Parker, M. Paterson, K.G. Beaty, M. Lyng \& S.E.M. Kasian. 2008. Eutrophication of lakes cannot be controlled by reducing nitrogen input: results of a 37 year whole ecosystem experiment. Proceedings of the National Academy of Sciences 105(11): 254-258.

Springer, J.J., J.M. Burkholder, P.M. Glibert \& R.E. Reed. 2005. Use of a real-time remote monitoring network and ship borne sampling to characterize a dinoflagellate bloom in the Neuse Estuary, North Carolina, USA. Harmful Algae 4: 533-551.

Stevenson, R.J. \& J.P. Smol. 2003. Use of algae in environmental assessments. In: J.D. Wehr \& R.G. Sheath (eds.), Freshwater algae of North America: ecology and classification. pp. 775-804. Academic Press, San Diego.

SigueE, D.C. 2005. Freshwater Microbiology: Biodiversity and dynamic interactions of microorganisms in the aquatic environment. Wiley \& Sons Ltd. Manchester. 525. pp.

Triemer, R.E. \& M.A. Farmer. 2007. A decade of euglenoid molecular phylogenetics. In: J. Brodie \& J. Lewis (eds.), Unravelling the algae: the past, present, and future of algal systematics. The Systematic Association, Special Volume Ser. 75, pp. 315-330. CRC Press.

UNESCO. 2009. Cianobacterias Planctónicas del Uruguay. Manual para la identificación y medidas de gestión. Bonilla, S. (ed.), Documento Técnico PHI-LAC, N 16. 105 pp.

UTERMÖHL, H. 1958. Zur vervollkommnung der quantitativen Phytoplankton Methodik. Mitteilungen Internationale Vereinigung für Theoretische und Angewandte Limnologie 9: 1-38.

Welch, E.B. 1992. Ecological effects of wastewater Applied Limnology and pollutant effects. Chapman \& Hall. 425 pp.

Welschmeyer, N. 1994. Fluorometric analysis of chlorophyll-a in the presence of chlorophyll-b and phaeopigments. Limnology and Oceanography 398: 1985-1992.

Xu, Y., G. WANG, W. YANG \& R. LI. 2010. Dynamics of the water bloom-forming Microcystis and its relationship with physicochemical factors in Lake Xuanwu (China). Environmental Science and Pollution Research 17: 15811590 .

Recibido: 29.02.16

Aceptado: 17.08.16 